\title{
Comparison of 90-90 passive hamstring stretching technique plus knee to chest position with 90-90 passive hamstring stretching alone in normal healthy individuals of age 18 to 25 years
}

\author{
Abhijeet Arun Deshmukh",", Sampada Sadhoo ${ }^{2}$, Shubhangi Meshram ${ }^{3}$, K. Vidya ${ }^{4}$ \\ ${ }^{1}$ Assistant Professor, ${ }^{2,3}$ Internee, ${ }^{4}$ Postgraduate Student, Dept. of Neuro-Physiotherapy, VSPM's College of Physiotherapy, Nagpur, \\ Maharashtra, India
}

*Corresponding Author:

Email: abhijeetashish84ad@gmail.com

\begin{abstract}
Hamstring flexibility plays an important role in physical fitness. Hamstring tightness is determined by knee extension deficit. Passive stretching is simple and found effective to improve muscle flexibility. In clinical settings, 90-90 passive stretching test is used whereas knee to chest position stretches proximal ends of hamstrings which leads to isolated hip flexion and causes all three muscles tendons of hamstrings to lengthen to a greater degree because lever arms are larger at the hip. The present study hypothesized that stretching both proximal and distal ends of hamstrings will be more effective than stretching distal end in full range alone. Therefore present study determines whether adding component like knee to chest position to 90-90 passive hamstring stretch enhances the flexibility. Total 200 young healthy individuals (100 Males, 100 Females) of age 18 to 25 years participated in study. Individuals with bilateral hamstring tightness (>20 degrees) were included. Randomly, on one side passive 90-90 stretching technique was applied (60 seconds)and on other side 90-90 passive hamstring technique (30 seconds) plus knee to chest passive stretch (30 seconds) was applied and compared between both genders. Active knee extension test was used as an outcome measure for calculating hamstring tightness angle. Stastically unpaired ttest was used for comparison between tests. Results showed highly significant increase $(\mathrm{p}=0.0005)$ in knee extension range of motion with 90-90 passive hamstring stretch plus knee to chest position than 90-90 hamstring stretch alone. Hence, 90-90 passive hamstring stretching technique plus knee to chest position found more effective in hamstring flexibility than $90-90$ passive hamstring stretch alone.
\end{abstract}

Keywords: Hamstrings stretching, Flexibility, 90-90 Hamstrings test, Knee to chest position, Active knee extension test.

\section{Introduction}

A proper stretching program is a key to achieve flexibility. ${ }^{1}$ Hamstring is one of the muscle group that have a tendency to shorten. ${ }^{2}$ Hamstring is a group of muscles including semitendinosus, semimembranosus, biceps femoris and it functions as flexor of knee joint and extensor of hip joint. $^{3}$ Muscle shortening usually results in individuals with low levels of physical activity, sedentary lifestyle, postural deviations, muscle imbalances and prolonged immobilization. Hamstring tightness has been reported to be the cause of posterior pelvic tilt which reduces lumbar lordosis and cause exacerbation of existing pain in low back. Hamstring tightness causes slight knee flexion during activities and inputs higher quadriceps forces to overcome the passive resistance of the hamstring, which may lead to increased patellofemoral joint reaction forces thus results in knee joint pain., ${ }^{4,5}$

Hamstring flexibility is an important component of physical fitness and plays a significant role in protecting the spine from possible risk. ${ }^{6}$ Decreased hamstring flexibility is a risk factor for development of hamstring strain injury in athletes. Hamstring flexibility is determined as knee extension deficit (KED). ${ }^{7}$ KED is determined by using active knee extension (AKET) which is having inter-rater reliability of 0.99 of both legs. Flexibility can be enhanced by many methods such as manual or mechanical/passive or assisted stretching, self-stretching, proprioceptive neuromuscular facilitation $(\mathrm{PNF})^{8}$ and inhibition techniques, joint mobilization/manipulation, muscle energy techniques ${ }^{9}$ soft tissue mobilization and manipulation and neural tissue mobilization (neuromeningeal mobilization). Out of these, passive stretching is a simple, nonsurgical procedure which includes lengthening of the shortened muscles and it is found that hamstring flexibility improves significantly when hamstring stretching exercises are performed systematically. ${ }^{10}$

Stretching is a general term used to describe any therapeutic maneuver designed to increase the extensibility of soft tissues. It is important to lengthen structures that have adaptively shortened and have become hypomobile overtime. Stretching exercises are thought to be an important element of fitness and conditioning programs designed to promote wellness. Stretching interventions can improve flexibility and range of motion. The effects of stretching intervention are reduction of pain and post exercises muscle soreness and it also improves physical performance. Stretching also helps in injury prevention or risk reduction and promotes proper posture while sitting or standing, improve mobility such as simply picking up a piece of paper off the floor and improve coordination. ${ }^{11,12}$ There is a hypothesis in the field of stretching, that increased flexibility after stretching may be attributed to an increase in muscle metabolism and hence temperature. Increasing muscle temperature will significantly decrease muscle stiffness, hence it is easy to handle the stress in muscles and strain in ligaments before injury. ${ }^{13}$

Different stretching techniques such as static stretching, ${ }^{14} \quad$ cyclic stretching, ${ }^{15}$ proprioceptive neuromuscular facilitation protocol $^{8}$ and ballistic stretching ${ }^{16}$ have been used to enhance flexibility in clinical 
setting. Static stretching is a commonly used method of stretching in which soft tissues are elongated just past the point of tissue resistance and then held in the lengthened position with a sustained stretch force over a period of 3060 seconds. ${ }^{2}$ Static stretching is the lengthening of muscle to its maximum range of motion while maintaining the position for a given amount of time. Static stretching is well accepted as an effective form of stretching to improve flexibility and has been considered a safer form of stretching. Commonly in the clinical settings, static stretching for tight hamstring is given in the form of 90-90 passive stretch. In this position, hip is flexed at 90 degrees and knee is completely extended, both of these motions contribute to hamstring stretch. ${ }^{17}$ Isolated hip flexion causes all three muscle tendon units to lengthen to a greater degree because lever arms are larger at the hip. ${ }^{18}$

In knee to chest position, there is full flexion of hip joint so that it touches to the chest where stretches proximal ends of hamstring because their lever arms are larger at the hip..$^{10,19}$ The present study hypothesized that stretching both proximal and distal ends of hamstring will be more effective than stretching distal end of hamstring in full range alone. It is important to determine which method is more effective in clinical settings that would help the physical therapist to choose the most effective hamstring stretching technique to improve general mobility of an individual and to prevent hamstring strain injuries. Therefore the aim of the study is to determine whether adding a component like knee to chest position, (that stretches proximal hamstring in full range) to 90-90 passive hamstring stretch enhances the flexibility.

\section{Materials and Methods}

A comparative experimental study was conducted on 200 normal healthy individuals (100 males and 100 females) between the age group of 18 to 25 years. Institutional ethics committee approval was obtained to carry out research work. A simple random sampling method was used to select the subjects in the study. The purpose of study was explained and written consent was obtained from subjects. The subjects were evaluated for bilateral hamstring muscle tightness and were determined by knee extension deficit (KED) by active knee extension test (AKET) (Fig. 1, 2).

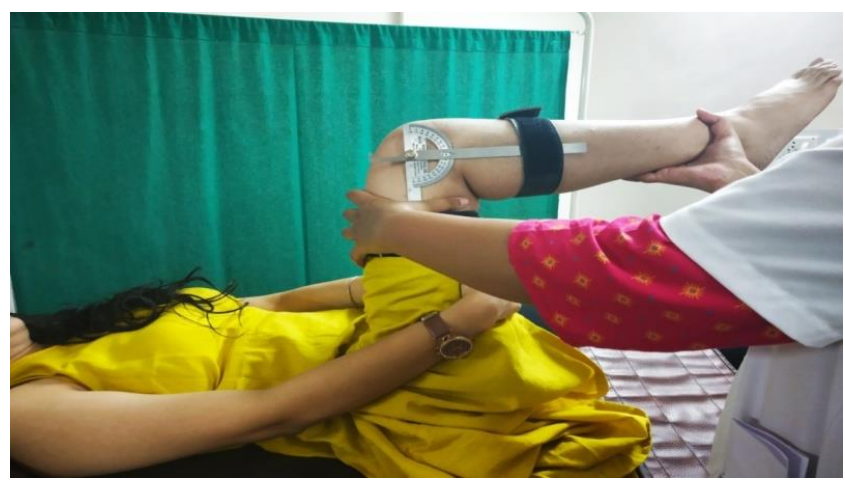

Fig. 1: Initial position of active knee extension test (AKET)

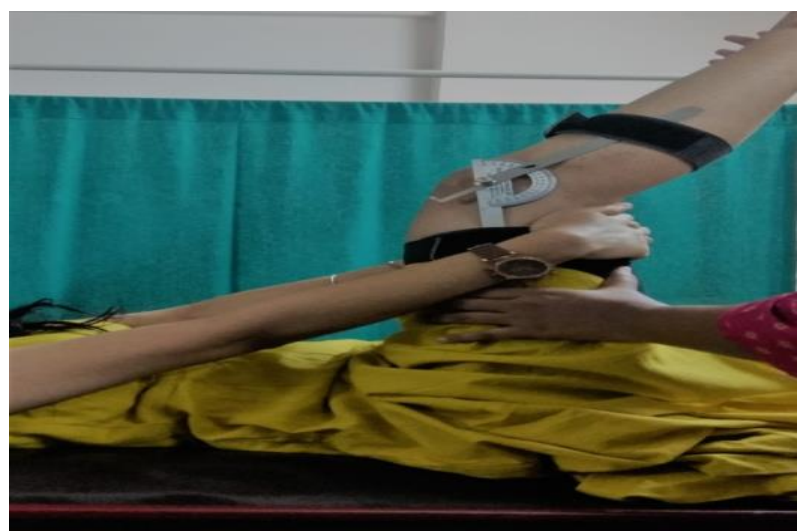

Fig. 2: End position of active knee extension test (AKET)

KED angle was measured by universal goniometer (Fig. 3 ). The subjects having bilateral hamstring tightness of 20 degrees or more were included in the study. Any history of low back pain within last 3 weeks, history of lower limb injury within last 6 weeks, hypermobility at knee joint/joints, history of hamstring tear, history of any orthopedic surgery of lower limb within last 6 weeks and subjects with any neurological disorder were excluded from the study. The study was conducted in duration of 4 months.

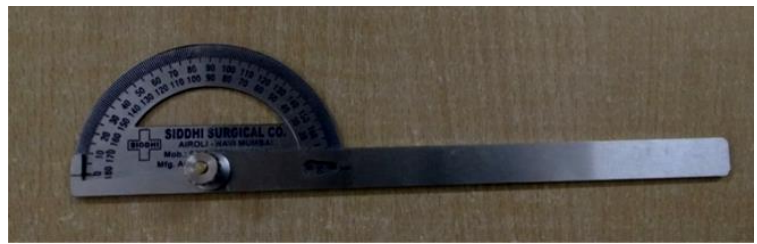

Fig. 3: Universal goniometer

The subject was lying in supine position on treatment table. On each subject, on left leg, 90-90 passive hamstring stretching (17) was performed for 60 seconds and on right leg, knee to chest position (Fig. 4) for 30 seconds plus $90-90$ passive stretching for 30 seconds was performed (2), duration of stretch was measured by using stopwatch (Fig. 5). Stabilizing belt was used to keep opposite thigh in neutral position (Fig. 6). No rest was allowed between each session on right leg. After one minute rest, post stretching hamstring flexibility was measured by AKET in degrees with the help of universal goniometer (Fig. 1, 2) $(20,21)$. The data was collected and analyzed.

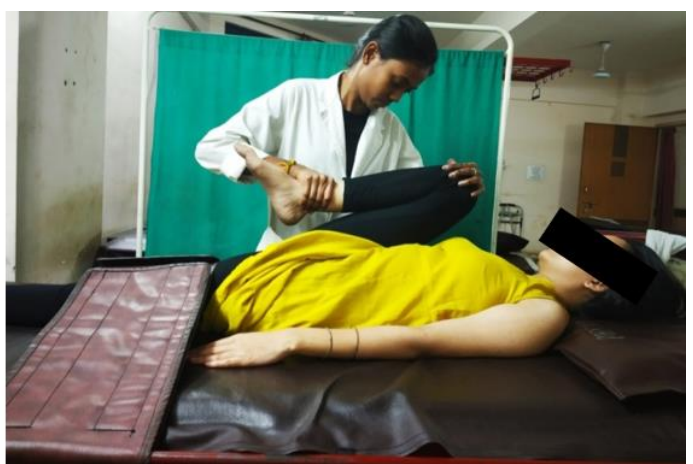

Fig. 4: Knee to chest position 


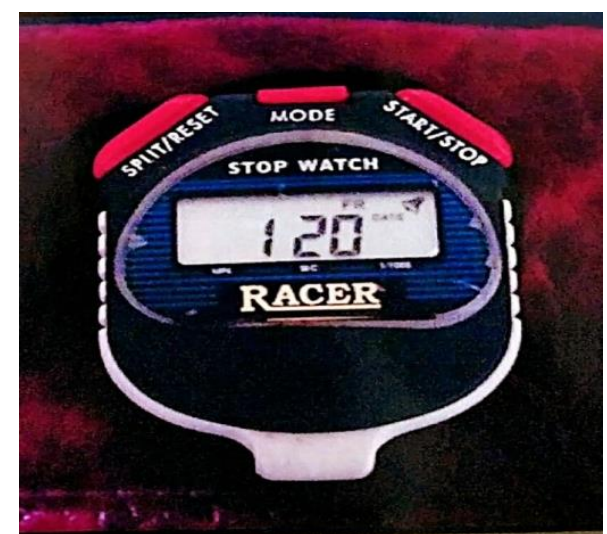

Fig. 5: Stopwatch

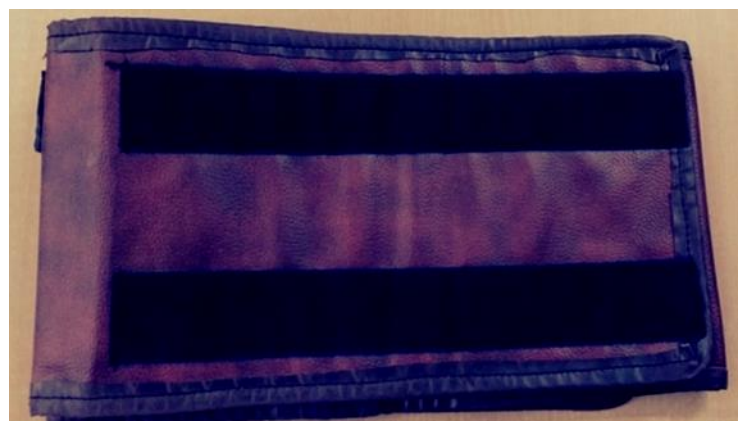

\section{Data Analysis}

The data was stastically analyzed using EPI info software W.H.O, version-3.4.3. Statistical unpaired -t-test was used to compare 90-90 passive stretching technique plus knee to chest position and 90-90 passive hamstring stretching alone within right and left leg and in both genders. Level of significance used $\mathrm{p}<0.05$.

\section{Results}

The distribution of individuals according to age is shown in Table 1. The mean of demographic data (height and weight) is shown in Table 2.

Fig. 6: Stabilizing belt

Table 1: Mean of demographic data (Age, gender, height and weight)

\begin{tabular}{|c|cc|c|c|}
\hline \multirow{2}{*}{ Age Group(years) } & Gender & n & Height(cm) $)^{\mathbf{a}}$ & Weight(kg) $^{\mathbf{a}}$ \\
\hline \multirow{3}{*}{$18-19$} & M & 19 & 168.52 & 63.84 \\
\cline { 2 - 5 } $20-21$ & F & 28 & 168.10 & 61.46 \\
\hline \multirow{3}{*}{$22-23$} & M & 30 & 166.43 & 62.53 \\
\cline { 2 - 5 } & F & 27 & 164.00 & 61.07 \\
\cline { 2 - 5 } $24-25$ & M & 29 & 168.24 & 63.10 \\
\cline { 2 - 5 } & F & 28 & 163.67 & 59.92 \\
\cline { 2 - 5 } & M & 22 & 163.04 & 57.31 \\
\hline
\end{tabular}

${ }^{\mathrm{a}}$ values represent mean

Table 2: Pre stretch and post stretch knee extension range of motion (in degrees) after 90-90 passive hamstring stretch in males, females and both genders

\begin{tabular}{|l|c|c|c|c|c|c|}
\hline & \multicolumn{2}{|c|}{ Male } & \multicolumn{2}{c|}{ Female } & \multicolumn{2}{c|}{ Combined } \\
\hline & Mean & S.D & Mean & S.D & Mean & S.D \\
\hline Pre & 21.8 & 4.91 & 21.71 & 4.72 & 21.75 & 4.8 \\
\hline Post & 14.03 & 4.47 & 13.85 & 4.41 & 13.94 & 4.43 \\
\hline
\end{tabular}

The means and standard deviations (SD) of pre stretch and post stretch knee extension range of motion (ROM) after 90-90 hamstring stretch in both genders are shown in Table 3. In males, mean pre stretch knee extension ROM was 21.8 \pm 4.91 degrees and mean post stretch knee extension ROM was $14.03 \pm 4.47$ degrees. In females, mean pre stretch knee extension ROM was 21.71 14.72 degrees and mean post stretch knee extension ROM was 13.85 \pm 4.41 degrees. The mean pre-stretch knee extension ROM in both males and females was $21.75 \pm 4.80$ degrees and mean post knee extension ROM was 13.94 \pm 4.43 degrees. 
Table 3: Pre stretch and post stretch knee extension range of motion (in degrees) after 90-90 passive hamstring stretch plus knee to chest position in males, females and both

\begin{tabular}{|c|c|c|c|c|c|c|}
\hline & \multicolumn{2}{|c|}{ Males } & \multicolumn{2}{c|}{ Females } & \multicolumn{2}{c|}{ Combined } \\
\hline Group & Mean & S.D. & Mean & S.D. & Mean & S.D. \\
\hline Pre & 24.77 & 4.94 & 25.03 & 6.16 & 24.9 & 5.57 \\
\hline Post & 16.02 & 4.75 & 15.79 & 6.12 & 15.91 & 5.47 \\
\hline
\end{tabular}

The mean and SD of pre stretch and post stretch knee extension ROM after 90-90 passive hamstring stretch plus knee to chest position are shown in Table 4. In males, mean pre stretch knee extension ROM was $24.47 \pm 4.94$ degrees and mean post stretch knee extension ROM was $16.02 \pm 4.75$ degrees. In females, mean pre stretch knee extension ROM was $25.03 \pm 6.16$ degrees and mean post stretch knee extension ROM was $15.79 \pm 6.12$ degrees. The mean pre stretch knee extension ROM in both males and females was $24.9 \pm 5.57$ degrees and mean post stretch knee extension ROM was $15.91 \pm 5.47$ degrees.

Table 4: Mean increase in knee extension range of motion after 90-90 passive hamstring stretch and 90-90 passive hamstring stretch plus knee to chest position in males

\begin{tabular}{|c|c|c|c|c|}
\hline Group & Mean & S.D. & t-value & p-value \\
\hline 90-90 passive hamstring stretch & 7.94 & 2.49 & \multirow[b]{2}{*}{2.2} & \multirow[b]{2}{*}{$0.028^{\mathrm{b}}$} \\
\hline 90-90 passive hamstring stretch plus knee to chest position & 8.85 & 3.29 & & \\
\hline
\end{tabular}
${ }^{\mathrm{b}} \mathrm{P} \leq 0.05$

The mean and SD of increase in knee extension ROM with 90-90 passive hamstring stretch and 90-90 passive hamstring stretch plus knee to chest position in males are shown in Table 5. Mean increase in knee extension ROM with $90-90$ passive hamstring stretch was $7.94 \pm 2.49$

degrees. Mean increase in knee extension ROM with 90-90 passive hamstring stretch plus knee to chest position was $8.85 \pm 3.29$ degrees. This shows significant increase in knee extension ROM with 90-90 passive hamstring stretch plus knee to chest position than $90-90$ passive hamstring stretch alone with $\mathrm{p}=0.028$ (statistically significant).

Table 5: Mean increase in knee extension range of motion after 90-90 passive hamstring stretch and 90-90 passive hamstring stretch plus knee to chest position in females

\begin{tabular}{|c|c|c|c|c|}
\hline Group & Mean & S.D. & t-value & p-value \\
\hline 90-90 passive hamstring stretch & 7.86 & 2.37 & & \\
\hline 90-90 passive hamstring stretch plus knee to chest position & 9.26 & 3.01 & 3.65 & $0.0003^{b}$ \\
\hline
\end{tabular}

${ }^{b} \mathrm{p} \leq 0.05,{ }^{b} \mathrm{p} \leq 0.001$

The mean and SD of increase in knee extension ROM with 90-90 passive hamstring stretch and 90-90 passive hamstring stretch plus knee to chest position in females are shown in Table 6. Mean increase in knee extension ROM with $90-90$ hamstring stretch was $7.86 \pm 2.37$ degrees. Mean

increase in knee extension ROM with 90-90 passive hamstring stretch plus knee to chest position was $9.26 \pm 3.01$ degrees. This shows highly significant increase in knee extension ROM with 90-90 passive hamstring stretch plus knee to chest position than $90-90$ passive hamstring stretch alone with $\mathrm{p}=0.0003$ (statistically high significant).

Table 6: Mean increase in knee extension range of motion after 90-90 passive hamstring stretch and 90-90 passive hamstring stretch plus knee to chest position in both males and females

\begin{tabular}{|l|c|c|c|c|}
\hline \multicolumn{1}{|c|}{ Group } & Mean & S.D. & t- Value & p- Value \\
\hline 90-90 passive hamstring stretch & 7.9 & 2.43 & & \\
\cline { 1 - 2 } plus knee to chest(passive) & 9.05 & 3.15 & 4.1 & $0.00005^{\mathrm{b}}$ \\
\hline
\end{tabular}

${ }^{\mathrm{a}} \mathrm{p} \leq 0.05,{ }^{\mathrm{b}} \mathrm{p} \leq 0.001$

The mean and SD of increase in knee extension ROM with 90-90 passive hamstring stretch and 90-90 passive hamstring stretch plus knee to chest position in both males and females showed mean increase in knee extension ROM with $90-90$ hamstring stretch was $7.9 \pm 2.43$ degrees. Mean increase in knee extension ROM with 90-90 passive hamstring stretch plus knee to chest position was $9.05 \pm 3.15$ degrees. This shows highly significant increase in knee extension ROM with 90-90 passive hamstring stretch plus

knee to chest position than 90-90 hamstring stretch alone with $\mathrm{p}=0.00005$ (statistically high significant).

\section{Discussion}

The aim of the present study was to compare $90-90$ passive hamstring stretching technique plus knee to chest position and 90-90 passive hamstring stretching technique alone. In the present study, the 90-90 passive hamstring stretching plus knee to chest position showed high significant increase in hamstring flexibility as compared to 90-90 passive hamstring stretch alone. Lack of hamstring 
flexibility contributes to injuries and weak hamstring (imbalance between strong quadriceps and weak hamstring) is highly susceptible for injuries. ${ }^{4,5}$ The best preventive measures involve a consistent program of both stretching and strengthening exercises of lower limb muscles and increase flexibility will contribute greatly to the ability of hamstring muscle to resist strains and injuries. ${ }^{22}$ Static stretching resulting in an increased flexibility due to changes in viscoelastic properties in the muscle fibers and this type of stretching may adjust the positional sensitivity of the Golgi tendon organs by affecting the series elastic component of the muscle. ${ }^{12}$

Chumanov ES (2011) and Wan X (2017) stated that, the hamstring lengthen during the late swing phase when hip is flexed and knee is extended since both of these motions contribute to hamstring stretch. ${ }^{17,23}$ In $90-90$ hamstring stretching technique, hip is flexed and knee is extended thus hamstrings get stretched. As proved by McIntyre E, isolated hip flexion causes lengthening of all three muscle tendon units to lengthen at hip, and in knee to chest position the hamstrings are lengthened proximally at the hip joint as the hip is fully flexed in this position. ${ }^{18}$

In the present study, significant increase in hamstring flexibility was achieved on the side performed 90-90 passive hamstring stretching technique plus knee to chest position than on the side performed $90-90$ passive hamstring stretching technique alone in both males and females. Initially the proximal hamstrings was maintained in lengthened position by knee to chest position and later the hamstring was also stretched at distal end in full range with knee extension, maintaining hip joint in 90 degrees of flexion which lead to achieve more knee extension range of motion. In the present study, females showed more increase in hamstring flexibility than males which is in agreement of previous study where it was found that females are relatively more flexible than men. ${ }^{24}$

In study (2017), it was found that similar hamstring muscle flexibility showed different maximal strain in a given movement and showed significant difference among both genders. ${ }^{25}$ It was believed that static stretching facilitates the Golgi tendon organ which produces an autogenic inhibition of muscle that is being stretched. Static stretching may be effective in increasing the length of muscle due to the prolonged stretching, which may allow the muscle spindle to adapt over time and cease firing. This adaptation/relaxation phenomenon of the muscle spindle contributes to an increase in length of the muscle. ${ }^{16}$ In the present study the duration of stretch was equal in both the stretching techniques, i.e. 60 seconds, which was proved to be equally effective with other duration of stretch. ${ }^{1,2}$ Hence it can be concluded that even though the duration of stretch was equal, 90-90 passive hamstring stretching plus knee to chest position showed significant increase in length as compared to 90-90 passive hamstring stretch alone. So it can be stated that stretching method had shown significant increase in muscle flexibility than duration of stretch.

\section{Conclusion}

Hamstring stretching is more effective if both proximal and distal ends are stretched in full range at both the joints. Hence it can be concluded that stretching with 90-90 passive hamstring stretching technique plus knee to chest position is more effective than 90-90 passive hamstring stretching alone.

\section{Implication for Future Research}

It can be recommended for future study that this concept of stretching both proximal and distal ends of muscles may give significant increase in flexibility in other two joint muscle of the body. (e.g. gastrocnemious, rectus femoris, iliopoas, long head of biceps etc). Long term effect of 90-90 passive hamstring stretching technique plus knee to chest position should be evaluated for hamstring flexibility in experimental study. The efficiency of this technique should be evaluated in all conditions which lead to hamstring tightness of moderate to severe intensity like spasticity in stroke, cerebral palsy, rigidity in Parkinson disease or in conditions of prolonged immobilization muscle tightness etc.

\section{Acknowledgement}

We are thankful and fortunate enough to get constant encouragement, support and guidance from all Teaching staffs of VSPM's College Of Physiotherapy which helped us in successfully completing our project work.

\section{Conflict of Interest: None.}

\section{References}

1. Fasen JM, O'Conner AM, Schwartz SL, Watson JQ, Plastaras CT, Garvan CW, et al. A randomized controlled trial of hamstring stretching; Comparison of four techniques. $J$ Strength Cond Res 2009;23(2):660-662.

2. Odunaiya NA, Hamzat TK, Ajay OF. The effects of static stretch duration on the flexibility of hamstring muscles. Afr $J$ Biomed Res 2005;8:79-82.

3. Kisner C, Colby LA. Therapeutic exercise: Foundations and Technique. 5th ed. New Delhi. India: Jaypee Brothers; 2007:65-67, 79.

4. Waryasz GR, McDermott AY. Patellofemoral pain syndrome (PFPS): a systemic review of anatomy and potential risk factors. Dyn Med 2008;7:9.

5. White LC, Dolphin P, Dixon J. Hamstring length in patellofemoral pain syndrome. Physiother 2009;95:24-28.

6. Decoster LC, Cleland J, Altieri C, Russell P. The effects of hamstring stretching on range of motion: A systemic literature review. J Orthop Sports Phys Ther 2005;35:377-387.

7. Gajdosik R, Lusin G. Hamstring muscle tightness: Reliability of an active-knee-extension test. Phys Ther 1983;63(7):10851088.

8. O'Hora J, Carwright A, Wade CD, Hough AD, Shum GLK. Efficacy of static stretching and proprioceptive neuromuscular facilitation stretch on hamstring muscle after a single session. $J$ Srength Cond Res 2011;25(6):1586-1591.

9. Ballantyne F, Fryer G, McLaughlin P. The effect of muscle energy technique on hamstring extensibility: the mechanism of altered flexibility. J Osteopath Med 2003;6(2):59-60. 
10. Rodriguez PL, Santoza FM, Lopez- Minaro PA, Baranda PS, Yuste JL. Effect of physical education stretching program on sit and reach score in school children. Sci Sports 2008;23(3): 170-175.

11. Waseem M, Nuhmani S, Ram CS, Ahmed F. A comparative study; static stretching versus eccentric training on popliteal angle in normal healthy individual collegiate males. Int J Sport Sci Eng 2009;3:180-186.

12. De Weijer VC, Gorniak GC, Shamus E. The effect of static stretch and warm-up exercise on hamstring length over the course of 24 hours. J Ortho Sport Phys Ther 2003;33(12):727733.

13. Slade B. The effect of static stretching on of sample of $18-25$ year-old students at Saint Martin's University. Saint Martin's University Biol J 2006:1:213-222.

14. Worrell TW, Smith TL, Winegardner J. Effect of hamstring stretching on hamstring muscle performance. J Orthop sport Phys Ther 1994;20(3):154-159.

15. Starring DT, Gossman MR, Nicholson GG, Lemons J. Comparison of cyclic and sustained passive stretching using a mechanical device to increase resting length of hamstring muscles. Phys Ther 1988;69(3):314-320.

16. Nelson RT, Bandy WD. Eccentric Training and Static Stretching Improve Flexibility of High School Males. J Athl Train 2004;39(3):254-259.

17. Chumanov ES, Heiderscheit BC, Thelen GG. Hamstring musculosketendon dynamics during stance and swing phases of high speed running. Med Sci Sport Enerc 2011;43(3):525532 .
18. McIntyre E, Ahmed A Oh Y, Kreinbrink J, Plmieri R, Wojtys E. On changes in hamstring length during a simulated jump landing: An in vitro study. Available on: www.asbweb.org/conferences/2006/pdfs/226.pdf.

19. Deville RJ. Two patients with a complete proximal rupture of the hamstring. Arch Orthop Trauma Surg 2010;130:523-526.

20. DePino GM, Webright WG, Arnold BL. Duration of maintained hamstring flexibility after cessation of an acute static stretching protocol. J Athl Train 2000;35(1):56-59.

21. Norkin CC, White DJ. Measurement of Joint Motion. $2^{\text {nd }}$ ed. New Delhi, India: Jypee Brothers; 1998:124,125,142,143.

22. Walker B. Hamstring injury treatment and hamstring stretching exercises. The stretching and sports Injury Newsletter .http://www.the stretchinghandbok.com.

23. Wan X. The effect of hamstring flexibility on peak hamstring muscle strain in sprinting. J Sport Health Sci 2017;6(3):283289.

24. Alter MJ. Science of flexibility. $3^{\text {rd }}$ ed.2004:120.

25. Wan X, Qu F, Garrett WE, Liu H, Yu B. Relationships among hamstring muscle optimal length and hamstring flexibility and strength. J Sport Health Sci 2017;6(3):275-282. 